\title{
Neoclassical transport simulations for stellarators
}

\section{$\operatorname{AUTHOR}(S):$}

Turkin, Y.; Beidler, C. D.; Maassberg, H.; Murakami, S.; Tribaldos, V.; Wakasa, A.

\section{CITATION:}

Turkin, Y. ... [et al]. Neoclassical transport simulations for stellarators. PHYSICS OF PLASMAS 2011, 18(2): 022505.

\section{ISSUE DATE:}

2011-02

URL:

http://hdl.handle.net/2433/160677

\section{RIGHT:}

Copyright 2011 American Institute of Physics. This article may be downloaded for personal use only. Any other use requires prior permission of the author and the American Institute of Physics. The following article appeared in PHYSICS OF PLASMAS 18, 022505 (2011) and may be found at http://link.aip.org/link/?php/18/022505 


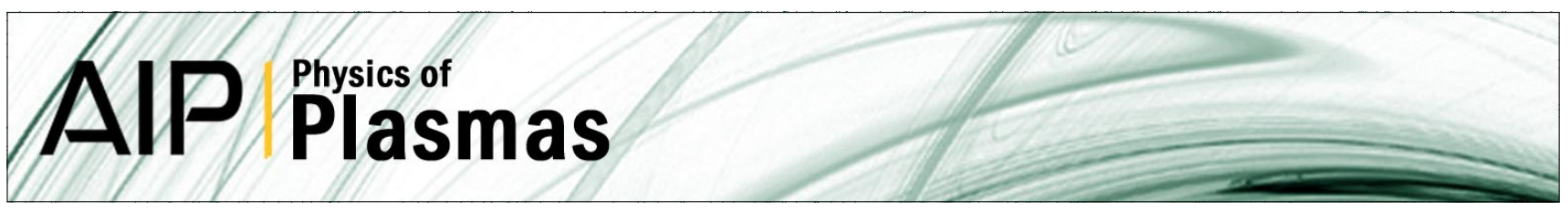

\section{Neoclassical transport simulations for stellarators}

Y. Turkin, C. D. Beidler, H. Maaßberg, S. Murakami, V. Tribaldos et al.

Citation: Phys. Plasmas 18, 022505 (2011); doi: 10.1063/1.3553025

View online: http://dx.doi.org/10.1063/1.3553025

View Table of Contents: http://pop.aip.org/resource/1/PHPAEN/v18/i2

Published by the American Institute of Physics.

\section{Related Articles}

Diagnostics development for quasi-steady-state operation of the Wendelstein 7-X stellarator (invited)

Rev. Sci. Instrum. 83, 10D730 (2012)

Effect of superbanana diffusion on fusion reactivity in stellarators

Phys. Plasmas 19, 082516 (2012)

The internal disruption as hard Magnetohydrodynamic limit of $1 / 2$ sawtooth like activity in large helical device Phys. Plasmas 19, 082512 (2012)

Internal disruptions and sawtooth like activity in Large Helical Device

Phys. Plasmas 19, 082501 (2012)

Zonal flows in stellarators in an ambient radial electric field

Phys. Plasmas 19, 072316 (2012)

\section{Additional information on Phys. Plasmas}

Journal Homepage: http://pop.aip.org/

Journal Information: http://pop.aip.org/about/about_the_journal

Top downloads: http://pop.aip.org/features/most_downloaded

Information for Authors: http://pop.aip.org/authors

\section{ADVERTISEMENT}

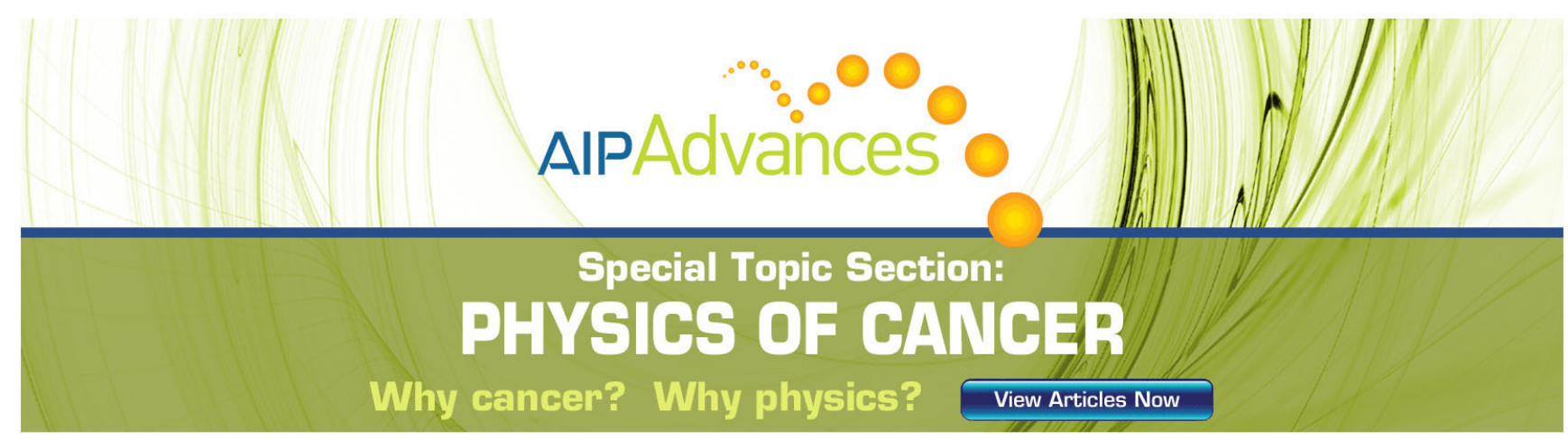




\title{
Neoclassical transport simulations for stellarators
}

\author{
Y. Turkin, ${ }^{1}$ C. D. Beidler, ${ }^{1}$ H. Maaßberg, ${ }^{1}$ S. Murakami, ${ }^{2}$ V. Tribaldos, ${ }^{3}$ and A. Wakasa ${ }^{2}$ \\ ${ }^{1}$ Max-Planck Institut für Plasmaphysik, EURATOM Association, D-17491 Greifswald, Germany \\ ${ }^{2}$ Department of Nuclear Engineering, Kyoto University, Kyoto 606-8501, Japan \\ ${ }^{3}$ Departamento de Física, Universidad Carlos III de Madrid, 28911 Leganés, Madrid, Spain
}

(Received 2 December 2010; accepted 14 January 2011; published online 9 February 2011)

\begin{abstract}
The benchmarking of the thermal neoclassical transport coefficients is described using examples of the Large Helical Device (LHD) and TJ-II stellarators. The thermal coefficients are evaluated by energy convolution of the monoenergetic coefficients obtained by direct interpolation or neural network techniques from the databases precalculated by different codes. The temperature profiles are calculated by a predictive transport code from the energy balance equations with the ambipolar radial electric field estimated from a diffusion equation to guarantee a unique and smooth solution, although several solutions of the ambipolarity condition may exist when root-finding is invoked; the density profiles are fixed. The thermal transport coefficients as well as the ambipolar radial electric field are compared and very reasonable agreement is found for both configurations. Together with an additional W7-X case, these configurations represent very different degrees of neoclassical confinement at low collisionalities. The impact of the neoclassical optimization on the energy confinement time is evaluated and the confinement times for different devices predicted by transport modeling are compared with the standard scaling for stellarators. Finally, all configurations are scaled to the same volume for a direct comparison of the volume-averaged pressure and the neoclassical degree of optimization. (C) 2011 American Institute of Physics. [doi:10.1063/1.3553025]
\end{abstract}

\section{INTRODUCTION}

Minimization of neoclassical transport is a key element in the design of economic fusion reactors based on the stellarator concept. In the long-mean-free-path (lmfp) regime, the neoclassical transport has a very unfavorable temperature dependence, particularly in the (electron) $1 / \nu$-regime for small radial electric fields where the neoclassical heat diffusivity scales as $T^{7 / 2}$. Although a detailed analysis of the experimental energy balance and its comparison with the neoclassical predictions is lacking for most devices, the W7-AS results clearly indicate that neoclassical confinement in the lmfp regime limits the achievable temperatures (see, e.g., Ref. 1). At low temperatures, however, neoclassical transport is less important due to the fairly strong temperature dependence of the radial transport coefficients. Furthermore, the measured radial electric fields are in rather good agreement with the prediction from the ambipolarity condition of the neoclassical particle fluxes. ${ }^{2}$

In the energy confinement time scaling ISSO $0,{ }^{3}$ a device (and even a configuration) dependent "renormalization" factor was introduced, describing the specific degree of energy confinement in the different devices (and configurations). With this factor included, the spread of all the experimental $\tau_{E}$ with respect to the scaling could be significantly reduced; however, the physical basis of this approach is still unclear. In this paper, the different levels of neoclassical confinement in a few stellarator examples (LHD, TJ-II, W7-AS, and $\mathrm{W} 7-\mathrm{X})$ are analyzed for high-performance scenarios in predictive transport simulations and compared with the ISS04 scaling.

Recently, a benchmarking of the three monoenergetic neoclassical transport coefficients with quite different numerical codes for the main stellarator configurations of interest has been completed (see Refs. 4 and 5). A very fast calculation of all thermal neoclassical transport coefficients by energy convolution can be performed based on interpolation in the databases of the monoenergetic coefficients for each magnetic configuration. This approach represents the most efficient means for neoclassical transport analysis of stellarator discharges since even the calculation of the monoenergetic coefficients requires significant computer resources, particularly at low collisionalities. The situation is similar for predictive neoclassical transport simulations.

In the traditional neoclassical ordering scheme, the five dimensional drift-kinetic equation is reduced to three dimensional by the local and monoenergetic ansatz, i.e., both the radius and the velocity appear only as parameters. In particular, energy diffusion is neglected and the simplified pitchangle collision operator violates parallel momentum conservation. However, parallel momentum correction techniques are available, ${ }^{6-9}$ which can be implemented in the energy convolution of the monoenergetic transport coefficients. Consequently, both the parallel conductivity and the bootstrap current are strongly affected by this parallel momentum correction (and also the radial neoclassical transport in tokamaks). In stellarators, however, the radial transport in the lmfp regime is dominated by the distribution function of the ripple-trapped particles, which is symmetric in the pitch, $p$ $=v_{\|} / v$, and is not affected by parallel momentum conservation. Consequently, the impact of momentum conservation on radial neoclassical transport in stellarators is negligible ${ }^{7,9}$ (except at very high collisionalities where the parallel viscous damping becomes very small).

The natural next step is the benchmarking of the thermal 
neoclassical transport coefficients and, in addition, the estimation of the ambipolar radial electric field. In this paper, the electron and ion energy balance equations are solved with a predictive transport code ${ }^{10}$ with neoclassical transport assumed (and "anomalous" transport contributions only at the outermost radii). These predictive transport simulations are based on interpolation in the databases of the monoenergetic coefficients precomputed with the DKES code. ${ }^{11,12}$ The benchmark is performed for a Large Helical Device (LHD) and a TJ-II vacuum configuration. Using (fixed) density and calculated temperature profiles for the LHD configuration, the thermal neoclassical transport coefficients are estimated with a neutral network interpolation (NNW) (Ref. 13) technique from a database of the monoenergetic coefficients precomputed with the Monte Carlo code DCOM. ${ }^{14,15}$ The radial electric field, $E_{r}$, is evaluated by root-finding from the ambipolarity condition of the neoclassical particle fluxes. The equivalent approach is performed for the TJ-II configuration also with a NNW technique and database results calculated by the Monte Carlo code MOCA. ${ }^{16}$ Then, the thermal neoclassical transport coefficients and the ambipolar radial electric fields are compared: the benchmarking is successful if reasonable agreement with the predictive simulations is obtained.

Equivalent predictive transport simulations are also performed for the W7-X vacuum configuration. These three configurations under investigation are characterized by a very different degree of neoclassical confinement optimization: a rather poor neoclassical confinement for the TJ-II configuration, a fairly good one for the LHD, and a very good one for the W7-X case. In the lmfp regime, the monoenergetic transport coefficients for $E_{r}=0$ at equivalent collisionalities differ by more than one order of magnitude, e.g., characterized by the effective helical ripple, $\epsilon_{\text {eff }}$, describing the radial transport in the $1 / \nu$-regime (see, e.g., Refs. 17 and 18). Due to the very strong nonlinearity of the thermal transport coefficients with respect to the temperatures, however, this difference in the neoclassical confinement is strongly reduced in global quantities such as the energy confinement time, $\tau_{E}$, and the volume-averaged $\langle\beta\rangle$. In particular, $\tau_{E}$ is compared with the confinement time scaling ISS04 (Ref. 3) in the predictive simulations for the three configurations. In addition, the three devices are scaled to the identical volume with fixed aspect ratio and identical magnetic field strength. This approach allows for a direct documentation of the impact of the different degree of neoclassical transport optimization as reflected in the global $\tau_{E}$ and $\langle\beta\rangle$ for identical heating power. Additionally, the required heating power to obtain the same $\langle\beta\rangle$ is also given for the three configurations.

In Sec. II, the neoclassical transport modeling is briefly summarized. Section III describes the benchmarking of the neoclassical thermal transport coefficients and of the ambipolar radial electric field for both the LHD and the TJ-II configurations; details on the diffusion equation for estimating $E_{r}$ compared with straightforward root-finding from the ambipolarity condition are given in Appendix A. In Sec. IV, a predictive transport simulation for the $\mathrm{W} 7-\mathrm{X}$ configuration is introduced, and all $\tau_{E}$ from III and IV are compared with the ISS04 scaling. Furthermore, the predictive transport simulations for the LHD and TJ-II configurations both scaled to the W7-X volume and for the same magnetic field strength are described. Finally, conclusions are given in Sec. V.

\section{BASICS OF NEOCLASSICAL MODELING}

Within the international collaboration on neoclassical transport in stellarators, ${ }^{4,5}$ the benchmark of the monoenergetic transport coefficients describing the radial transport, the bootstrap current, and the parallel conductivity has been successfully completed. As an outcome of these efforts, neoclassical databases have been created for several stellarators using various approaches including Monte Carlo simulations $^{13,16}$ and numerical solutions of the rippleaveraged ${ }^{19}$ and drift kinetic equations. ${ }^{12}$ These coefficients depend on the flux surface label and two dimensionless parameters: the normalized radial electric field and collisionality. However, in order to use the monoenergetic coefficients in the predictive transport codes and for the analysis of experimental results, the full neoclassical (or thermal) transport matrix must be obtained through database interpolation and appropriate convolutions of the resulting quantities with a local Maxwellian [see Eq. (4)]. The power balance of the plasma in the predictive transport code is described by equations for the electron and ion energies,

$$
\frac{3}{2} \frac{\partial n_{\alpha} T_{\alpha}}{\partial t}+\frac{1}{V^{\prime}} \frac{\partial}{\partial r} V^{\prime}\left(q_{\alpha}+\Gamma_{\alpha} T_{\alpha}-\chi_{\alpha} n_{\alpha} T_{\alpha}^{\prime}\right)=P_{\alpha}+Z_{\alpha} \Gamma_{\alpha} E_{r},
$$

where $P_{\alpha}$ is the power source term that includes the heating power, the bremsstrahlung by electrons, and the term describing the collisional coupling between electrons and ions. The neoclassical particle $\Gamma_{\alpha}$ and energy $q_{\alpha}$ fluxes are given by the following expressions:

$$
\begin{aligned}
& \Gamma_{\alpha}=-n_{\alpha} D_{1}^{\alpha}\left\{\left(\frac{n_{\alpha}^{\prime}}{n_{\alpha}}-\frac{Z_{\alpha} E_{r}}{T_{\alpha}}\right)+\left(\frac{D_{2}^{\alpha}}{D_{1}^{\alpha}}-\frac{3}{2}\right) \frac{T_{\alpha}^{\prime}}{T_{\alpha}}\right\}, \\
& q_{\alpha}=-n_{\alpha} T_{\alpha} D_{2}^{\alpha}\left\{\left(\frac{n_{\alpha}^{\prime}}{n_{\alpha}}-\frac{Z_{\alpha} E_{r}}{T_{\alpha}}\right)+\left(\frac{D_{3}^{\alpha}}{D_{2}^{\alpha}}-\frac{3}{2}\right) \frac{T_{\alpha}^{\prime}}{T_{\alpha}}\right\},
\end{aligned}
$$

with

$$
\begin{aligned}
& D_{n}^{\alpha}=\frac{2}{\sqrt{\pi}} \int_{0}^{\infty} \mathrm{d} K_{\alpha} \mathrm{e}^{-K_{\alpha}} K_{\alpha}^{n-0.5} D_{11}, \quad K_{\alpha}=\frac{m_{\alpha} v_{\alpha}^{2}}{2 T_{\alpha}}, \\
& D_{11}=D_{11}\left(r, \frac{\nu_{\alpha}}{v_{\alpha}}, \frac{E_{r}}{v_{\alpha} B_{0}}\right),
\end{aligned}
$$

where $m_{\alpha}, \nu_{\alpha}, v_{\alpha}, n_{\alpha}, T_{\alpha}, \chi_{\alpha}$, and $Z_{\alpha}$ are the mass, collision frequency, velocity, density, temperature, anomalous energy diffusivity, and charge number of electrons or ions, $E_{r}$ is the radial electric field, $B_{0}$ is the average value of magnetic field, the prime denotes the partial derivative with respect to the effective radius $r=\sqrt{\Phi / \pi B_{0}}$ with $\Phi$ being the toroidal magnetic flux, and $V$ is the volume inside the flux surface labeled by $r$. The diffusion coefficients $D_{n}^{\alpha}$ appearing in the fluxes are the result of energy convolution of the radial monoener- 

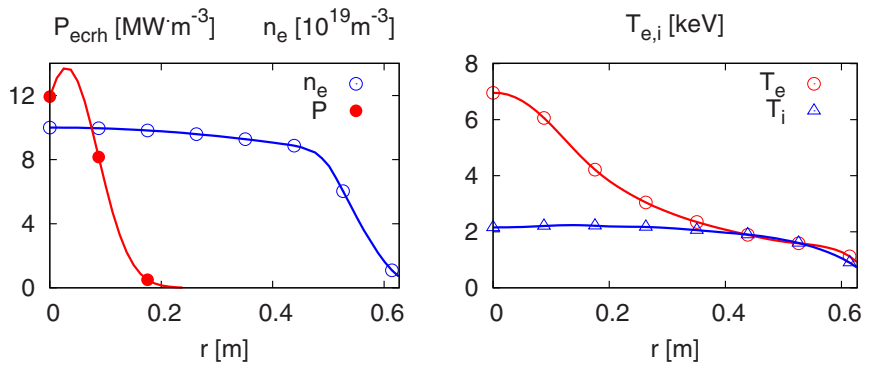

FIG. 1. (Color online) Plasma profiles for the inward-shifted LHD: density and ECRH power deposition profiles (left); predicted electron and ion temperatures (right).

getic transport coefficient $D_{11}$ with the Maxwellian distribution function.

Usually, the radial electric field $E_{r}$ is obtained by solving the ambipolarity constraint

$$
Z_{i} \Gamma_{i}=\Gamma_{e}
$$

In some cases, however, this approach suffers from the drawbacks of multiple roots and a discontinuity in dependence on the radial coordinate. These problems may be avoided by solving a partial differential equation for the radial electric field, which has the form of a diffusion equation,

$$
\frac{\partial E_{r}}{\partial t}-\frac{1}{V^{\prime}} \frac{\partial}{\partial r} D_{E} V^{\prime} r \frac{\partial}{\partial r} \frac{E_{r}}{r}=\frac{|e|}{\epsilon}\left(\Gamma_{e}-Z_{i} \Gamma_{i}\right),
$$

where $D_{E}$ is the diffusion coefficient of the electric field, which originates from the poloidal plasma viscosity (here, for simplicity, we assume that $D_{E}$ does not depend on $E_{r}$ ), and $\epsilon$ is the dielectric constant. This equation follows from the thermodynamic approach ${ }^{20-22}$ by minimizing the total heat production (A1) due to both the poloidal sheared rotation and the neoclassical transport (see Appendix A for more details). An equation similar to Eq. (7) can be derived using the higher order expansion of the distribution function within the neoclassical theory. ${ }^{23}$ A method for estimating the diffusion coefficient $D_{E}$ can be also found in Ref. 23 .

\section{BENCHMARKING OF THERMAL NEOCLASSICAL TRANSPORT}

In this section, we present benchmarking results of two techniques used for creating the full neoclassical transport matrix. The testing procedure is as follows. At first, we perform a "theoretical" experiment for LHD by applying the predictive transport code ${ }^{10}$ with neoclassical transport provided by the convolution based on the database created from DKES simulations of this device and conventional interpolation on the domain of three parameters: the plasma minor radius, the normalized radial electric field, and collisionality.

Then, the resulting plasma profiles are analyzed by means of the neoclassical database DCOM/NNW, which uses the Monte Carlo code DCOM for creating the discrete data set and neural network technique for interpolation during convolutions with a local Maxwellian. The outcome of transport analysis, the derived thermal diffusion coefficients and the ambipolar radial electric field, are compared with the original prediction. The same method is applied for the comparison of the predicted transport coefficients for the TJ-II stellarator with the ones resulting from transport analysis based on the MOCA database and neural network technique for interpolation of the database results.

\section{A. Prediction and benchmarking results for LHD}

The modeling has been performed by a transport code ${ }^{10}$ for a pure hydrogen plasma of $10^{20} \mathrm{~m}^{-3}$ density for the inward-shifted vacuum configuration of LHD with a major radius of $3.60 \mathrm{~m}$. The density profile in Fig. 1 is held fixed during the simulation and $10 \mathrm{MW}$ electron cyclotron resonance heating $(\mathrm{ECRH})$ is modeled by the Gaussian profile in Fig. 1. The transport model is chosen to be largely neoclassical; only near the plasma edge, where the neoclassical fluxes may strongly decrease due to the low temperature, a simple anomalous energy diffusivity $\chi_{e, i} \propto 1 / n_{e}$ is used to stabilize the numerical scheme (see Fig. 2). The neoclassical transport is provided by the convolution module based on the data created from DKES simulations of this device. The data
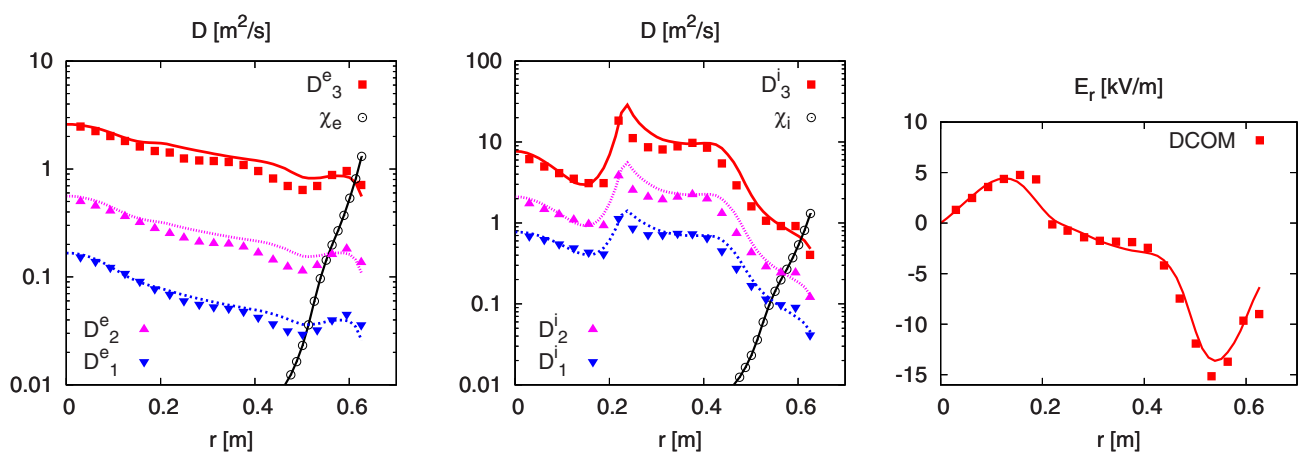

FIG. 2. (Color online) The thermal diffusion coefficients for electrons and ions (left and middle); the ambipolar radial electric field (right). Shown by lines are those from the original prediction; solid symbols are the results of analysis by DCOM/NNW; open circles denote anomalous energy diffusivity used in the predictive simulation. 

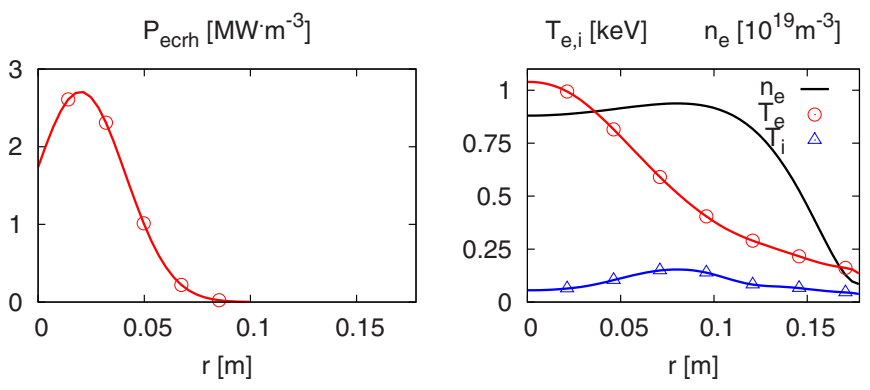

FIG. 3. (Color online) Plasma profiles for the TJ-II: electron heating profiles (left); the density and the predicted electron and ion temperatures (right).

file contains approximately 3000 records of monoenergetic coefficients stored for a number of radial positions, normalized electric fields, and collisionalities. The interpolation is done by conventional techniques using asymptotic behavior when needed. Predicted temperatures are shown in Fig. 1.

The plasma profiles shown in Fig. 1 are used in the transport analysis performed by TASK3D (Ref. 24) with the neoclassical database DCOM/NNW. The results of both convolution techniques for evaluating the thermal diffusion coefficients are compared in Fig. 2.

The ambipolar radial electric field and thermal diffusion coefficients from both approaches coincide well. Some discrepancies are seen, especially where the ambipolar electric field changes rapidly. This can be explained by the different methods used for determining the ambipolar radial electric field. In the original prediction, the electric field is obtained from the diffusion equation (7), while in the analysis by DCOM/NNW, the electric field is found by solving the ambipolarity constraint (6) for the neoclassical radial particle fluxes of the electrons and ions. The electron diffusion coefficients have some disagreement in the region of strong density gradient. The ion diffusion coefficients are mainly affected in the region, where a transition occurs from the positive radial electric field to the negative one, i.e. from the "electron root" solution ${ }^{25}$ of the ambipolarity condition (6) to "ion root." However, the entire results demonstrate good agreement despite the quite different techniques used.

\section{B. Prediction and benchmarking results for TJ-II}

The prediction for TJ-II has been made by the transport code $^{10}$ for a pure hydrogen plasma of $10^{19} \mathrm{~m}^{-3}$ density for the standard magnetic configuration. The density profile in Fig. 3 is held fixed during the simulation and $300 \mathrm{~kW}$ electron heating is modeled by the Gaussian profile in Fig. 3. The anomalous transport model shown by the open circles in Fig. 4 is the same as in the LHD case. The neoclassical transport is provided by the convolution module that uses the data created from DKES runs. The results of the simulation, the electron and ion temperatures, are shown in the right portion of Fig. 3.

The predicted plasma profiles shown in Fig. 3 are used in the transport analysis based on the neoclassical database of monoenergetic coefficients created by MOCA runs. The results of analysis and the original prediction for the thermal diffusion coefficients and the radial electric field are compared in Fig. 4. The predicted radial electric field from the solution of the diffusion equation (7) is shown by the solid line in the right-hand plot of Fig. 4. The solutions of the ambipolarity constraint (6) using the predictive transport $\operatorname{code}^{10}$ shown by open squares and that from the MOCA based analysis code demonstrate good agreement within $r<0.11 \mathrm{~m}$; for the larger radii, the electron root branch has more pronounced mismatch. To calculate the thermal diffusion coefficients in the analysis code (MOCA based), the radial electric field from the original transport simulation is used (solid line in Fig. 4 right) because the electric field in the original prediction is obtained from the diffusion equation (7), while in the analysis code the electric field is the solution of the ambipolarity constraint (6) and in this case the special procedure to select roots is required (see Appendix A). The thermal diffusion coefficients from both approaches coincide well in the central portion of the plasma within $0.03<r<0.17 \mathrm{~m}$. However, rather big discrepancies are found for $r<0.03 \mathrm{~m}$. This can be explained by the different methods used for the extrapolation of the radial monoenergetic diffusion coefficient $D_{11}$ toward the magnetic axis, where both databases from DKES and MOCA runs
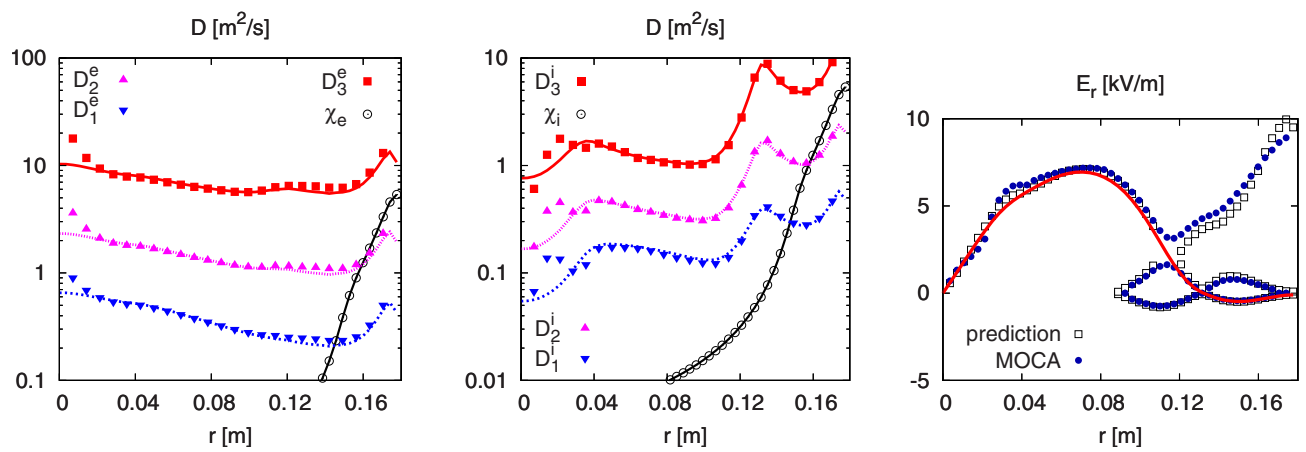

FIG. 4. (Color online) The thermal diffusion coefficients for electrons and ions (left and middle); the ambipolar radial electric field (right). Results from the original prediction are given by curves; solid symbols are the results of analysis by MOCA; open circles denote the anomalous energy diffusivities used in the predictive simulation; open squares (figure in right) are the roots of the ambipolarity condition (6) produced by applying the neoclassical module from the transport code (Ref. 10). 

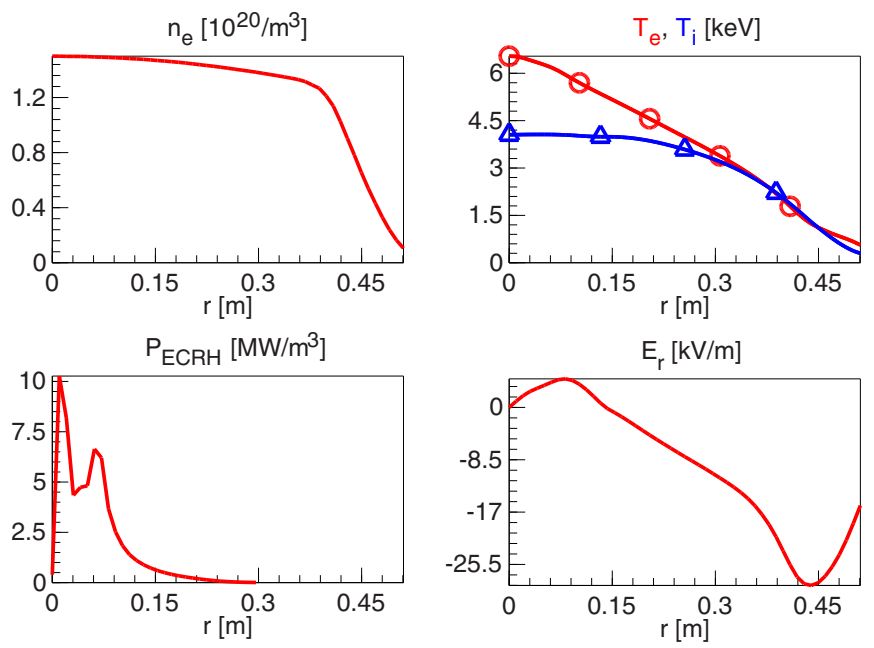

FIG. 5. (Color online) The W7-X plasma profiles for the 8 MW O2-mode heating scenario: assumed density (top left); predicted electron (circles) and ion (triangles) temperatures (top right); power deposition profile produced by ten ECRH beams in three path absorption scheme (bottom left); the radial electric field (bottom right).

contain no data. Again, as in the case of LHD, the entire results demonstrate good agreement despite the quite different techniques used.

\section{IMPACT OF NEOCLASSICAL TRANSPORT OPTIMIZATION}

First, a predictive transport simulation for the W7-X vacuum ("standard") configuration is described. Then, this W7-X configuration is used as a reference case to demonstrate the impact of neoclassical confinement optimization on global parameters such as $\tau_{E}$ and $\langle\beta\rangle$. For this purpose, the LHD and TJ-II configurations are scaled to the same volume as the W7-X case and this scaling is also applied to the corresponding DKES databases [no new DKES calculations are required (see Appendix B)].

\section{A. Transport simulations for a W7-X configuration}

The aim of this W7-X simulation is the analysis of a high- $\langle\beta\rangle$ discharge at full magnetic field $(2.5 \mathrm{~T})$. Consequently, a high density is assumed, which requires ECRH in O2-mode ${ }^{26,27}\left(n_{e}\right.$ is above the cutoff for the X2-mode) in order to improve confinement. An ECRH power of $8 \mathrm{MW}$ is assumed in this simulation, which will be the maximum ECRH power in the initial operation phase of W7-X. To be consistent with the LHD and TJ-II scenarios, also the W7-X vacuum case is used here.

Figure 5 shows an example of a multipass O2-mode heating scenario at $8 \mathrm{MW}$ ECRH provided by ten beams for $n_{e}=1.5 \times 10^{20} \mathrm{~m}^{-3}$. The transport model is chosen to be largely neoclassical; the electron and ion anomalous transport coefficients with an edge value of $1 \mathrm{~m}^{2} / \mathrm{s}$ are used only within the region of high density gradient (similar to the LHD case in Fig. 2) and have the form $\chi_{e, i} \propto h(r / a-1) / n_{e}$, where $h(x)=1+\tanh (4 x)$ and $a$ is the plasma minor radius. The resulting volume-averaged $\langle\beta\rangle$ is about $4.5 \%$. The energy confinement time $\tau_{E}=0.56 \mathrm{~s}$ is higher than that of
ISS04 scaling ${ }^{3}$ with an improvement factor of 3 , which reflects the very good neoclassical optimization (see also Fig. 8). The main portion of the ECRH power is absorbed after two passes; the overall absorption efficiency along three passes is slightly more than $98 \%$. A small electron root with a positive value of the radial electric field is obtained within the central ECRH deposition zone.

\section{B. The magnetic configurations}

The LHD magnetic configurations are of the $l=2$ heliotron type. ${ }^{28}$ In the central region, the rotational transform, $t$, is fairly flat and increases strongly at the outer radii. An inward or outward shift of the magnetic configuration has a large impact on the neoclassical confinement, which can be characterized in the $\operatorname{lmfp}$ regime by the effective ripple, $\epsilon_{\mathrm{eff}}$ (which corresponds to the helical ripple for a classical stellarator configuration). The LHD configuration used here in the benchmarking is inward-shifted with a major radius $R=3.60 \mathrm{~m}$. Since only the component of $\nabla B$ in the helical direction determines the bounce-averaged radial $\nabla B$ drift, deeply trapped particles are well confined (see Fig. 6). This inward-shifted LHD configuration is drift optimized ${ }^{15,29}$ (in the sense of " $\sigma$-optimization" $" 30$ ). Consequently, $\epsilon_{\text {eff }}$ is much smaller than the dominant helical ripple (defined by the $B_{21} / B_{00}$ Fourier mode) in this LHD configuration. This optimization, however, is lost at finite $\langle\beta\rangle$ (Ref. 13) but can be (at least partially) recovered by an increased inward shift.

TJ-II is a flexible heliac with bean-shaped flux surfaces rotating around the central conductor and with a fairly flat $t$ profile. The $B_{m n}$ Fourier spectrum for the TJ-II configurations is very broad due to the local minima in $B$, which reflect the coil ripple (see Fig. 6). The deeply trapped particles in the local ripples dominate the radial transport and lead to a rather poor neoclassical confinement, which is reflected in very large $\epsilon_{\text {eff }}$ values (see Fig. 7). For these conditions, additional nonlocal convective transport contributions $^{31}$ can further degrade confinement. It should be mentioned in this context that the original goal of TJ-II was MHD-stability investigations at high $\beta$ (Ref. 32) and not neoclassical confinement in the $1 \mathrm{mfp}$ regime. Due to the high $t(t \simeq 3 / 2$ for the standard configuration considered in this benchmarking), the Shafranov shift is rather small and $\beta$-effects on the neoclassical confinement are less important.

The W7-X standard configuration used here as the reference (the same current in all modular coils, no current in the planar ones) has very good neoclassical confinement, but neither the bootstrap current nor fast particle losses ( $\alpha$-particles in an equivalent reactor configuration) are sufficiently minimized to make this configuration an attractive reactor candidate in the sense of multiple optimization criteria, ${ }^{33}$ which should be simultaneously realized. Only with a rather large toroidal mirror term can the bootstrap current be reduced, but the price to pay is degraded confinement. The W7-X configuration selected here is probably not a candidate for quasistationary discharge scenarios, but promises high $\langle\beta\rangle$ at full magnetic field $(B=2.5 \mathrm{~T})$ with a realistic heating power level. The very strong elongation (reduction of the toroidal curvature term $B_{10}$ ) of all W7-X con- 

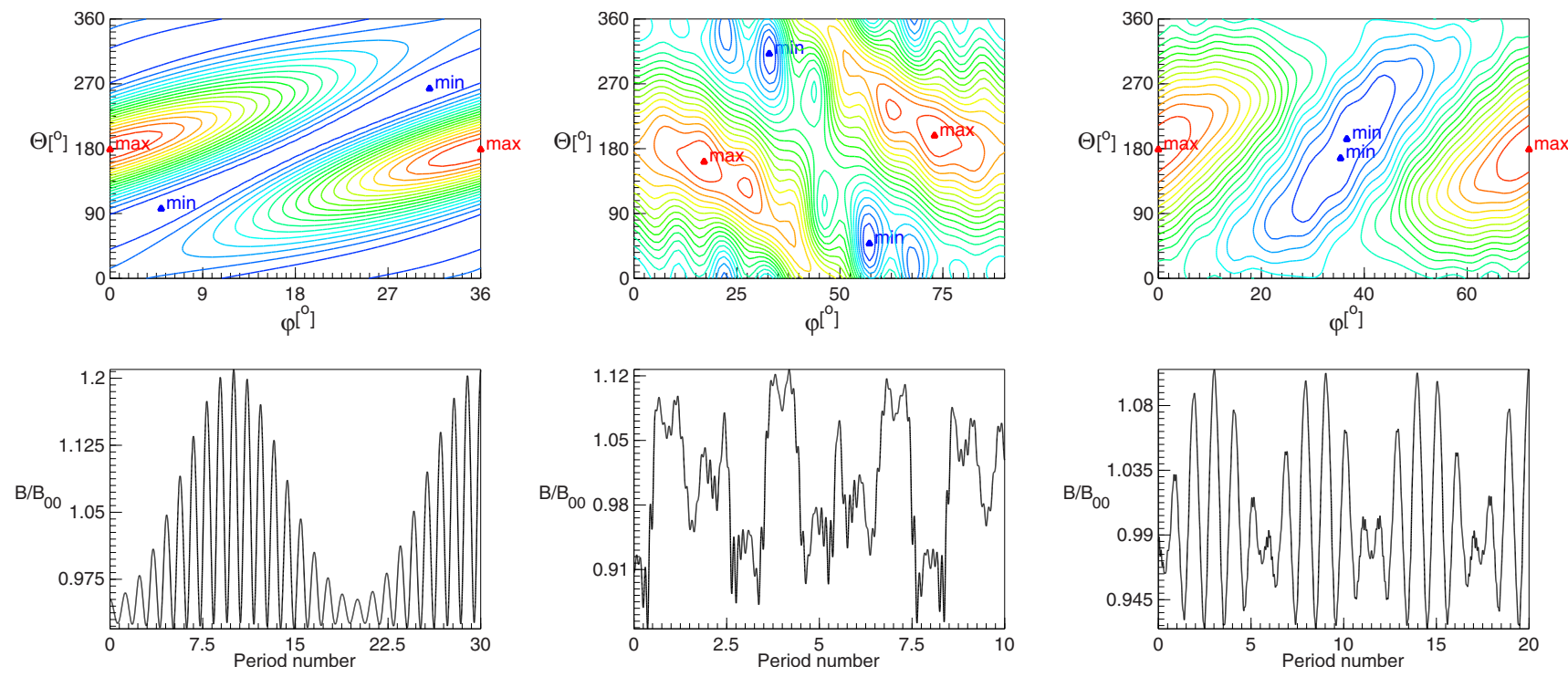

FIG. 6. (Color online) $|\mathbf{B}|$ for the flux surface at $r / a=0.5$ for LHD, TJ-II, and W7-X (from left to right); shown in the upper row is a contour plot of $|\mathbf{B}|$ on the flux surface; shown in the lower row is $|\mathbf{B}| / B_{00}$ along the field line.

figurations is an essential ingredient for the rather good neoclassical confinement. Finite $\beta$ leads, in general, to an additional reduction of the neoclassical transport, but increases the bootstrap current.

Figure 7 shows the effective ripple, $\epsilon_{\text {eff }}$, versus radius for the three configurations under consideration. The radial transport coefficient in the low collisional $1 / \nu$-regime with $E_{r} \simeq 0$ scales with $\epsilon_{\mathrm{eff}}^{3 / 2}$. Consequently, the confinement in this regime is different by more than one order of magnitude for the three configurations. In addition, the reduction of the averaged toroidal curvature is also given for reference.

The transport simulations described above are very different with respect to the volume, the magnetic field strength, the assumed density, and the heating power. Consequently, a direct comparison of the energy confinement is not possible and the ISSO4 scaling ${ }^{3}$ is used for reference. In density and power scans for W7-AS ECRH discharges with electrons dominating the energy flux, a rather good agreement of the experimental $\tau_{E}$ scaling with a purely neoclassical transport model for the $1 / \nu$-regime was found (see Fig. 3 in Ref. 34), which is in conflict to the ISS04 scaling. The situation becomes different if the ions dominate the energy flux. First predictive transport simulations for the W7-X standard con-
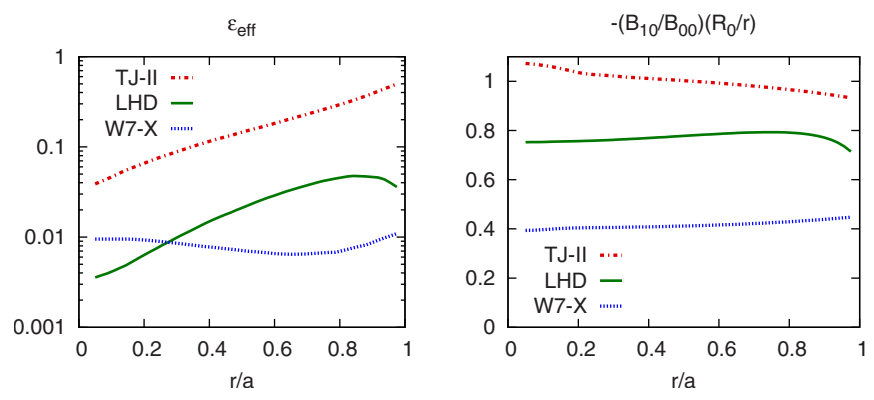

FIG. 7. (Color online) On the left, the effective helical ripple for $1 / \nu$ transport is shown as a function of the normalized minor radius; on the right, the measure of the elongation (normalized toroidal curvature term). figuration with both density and power scans (for ECRH, p-NBI, and n-NBI) follow roughly the $\tau_{E}$ of ISS04 but with improvement factors of $2-3,{ }^{34}$ which reflects the very good neoclassical confinement.

In Fig. 8, the experimentally found energy confinement times and the results of neoclassical simulations for $\mathrm{W} 7-\mathrm{X}$, LHD, W7-AS, and TJ-II are compared with the ISS04 $\tau_{E}$ scaling. Simulation of the W7-AS high-performance discharge demonstrates good agreement with the corresponding experimental result. The LHD simulation (Sec. III) exhibits a $\tau_{E}$ value about 1.4 times higher than expected from ISS04; this result manifests good neoclassical optimization of the

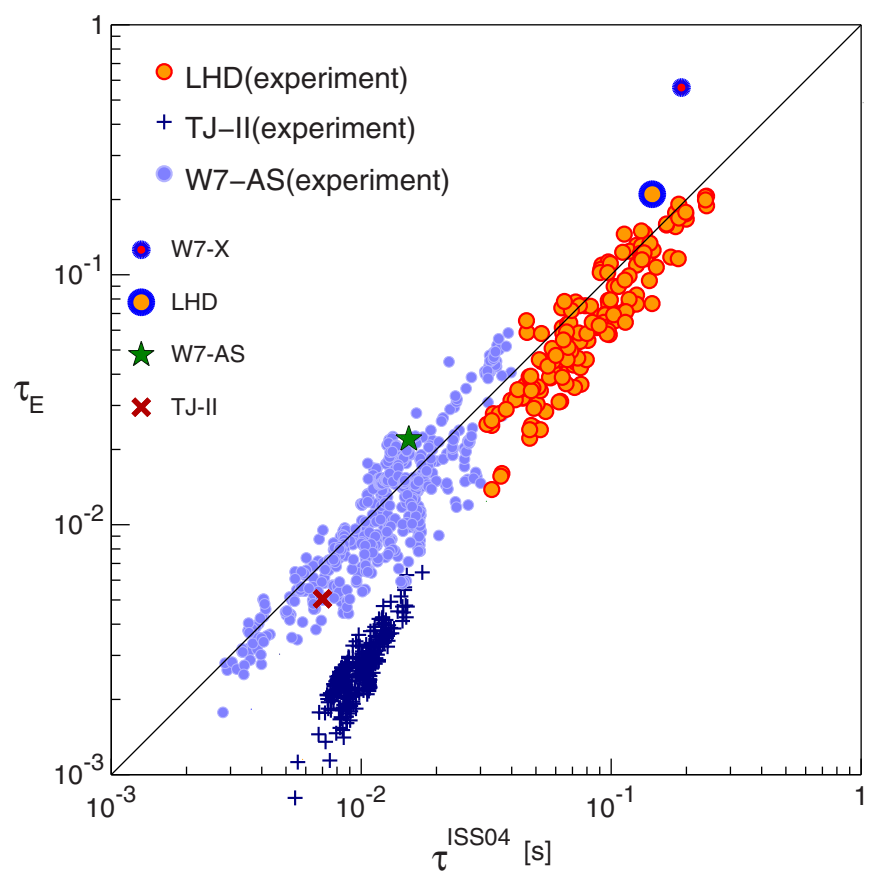

FIG. 8. (Color online) Experimentally found energy confinement times and results from neoclassical simulations vs ISS04 $\tau_{E}$ scaling. 


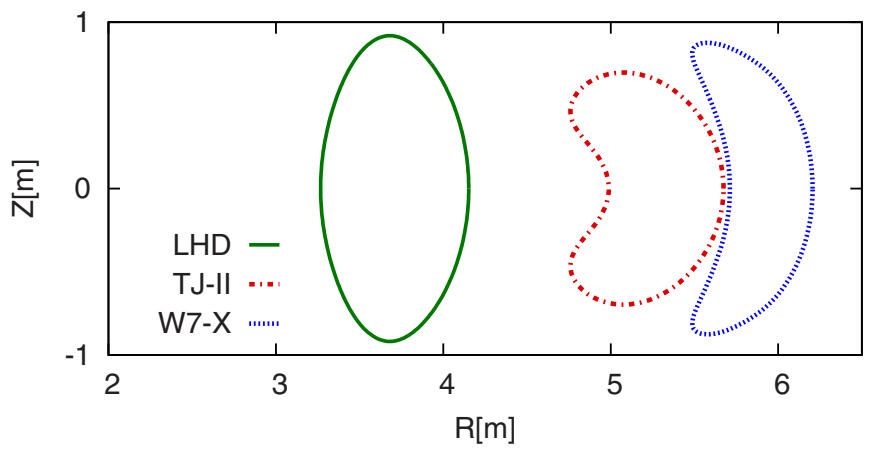

FIG. 9. (Color online) The last closed magnetic surface of the devices scaled to the volume of W7-X.

inward-shifted vacuum configuration of LHD. The $\tau_{E}$ from the TJ-II simulation (Sec. III) exhibits much better confinement than experimentally observed but nevertheless worse than ISS04 scaling. The energy confinement time for the W7-X example of $8 \mathrm{MW}$ ECRH in O2-mode with $n_{e}=1.5$ $\times 10^{20} \mathrm{~m}^{-3}$ is three times higher than the ISS04 prediction.

\section{Comparison of neoclassical confinement}

A direct comparison of the impact of neoclassical confinement optimization on the achievable $\langle\beta\rangle$ and $\tau_{E}$ can be performed with the different magnetic configurations scaled to the same volume while holding the aspect ratio, $R / a$, fixed (where $R$ and $a$ are the major and minor radii, respectively). All monoenergetic neoclassical transport coefficients for configurations scaled in size can be easily derived from the original database (see Appendix B for more details). Here, the standard W7-X vacuum configuration is used as the reference case. The inward-shifted LHD configuration has nearly the same volume (with a size scaling factor of $f_{s}=0.997$ ), whereas the TJ-II configuration must be increased by $f_{s}=3.074$. The cross section of the scaled LHD and TJ-II configurations together with the $\mathrm{W} 7-\mathrm{X}$ reference case are shown in Fig. 9. This size scaling factor $f_{s}$ is used in the transform of the monoenergetic transport coefficients in Eq. (B2). In addition, the magnetic field strength of $B=2.5 \mathrm{~T}$ and the same density profile with $n_{e}(0)=10^{20} \mathrm{~m}^{-3}$ are used for the predictive transport simulations in which an (artificial) central ECRH power deposition profile (with Gaussian shape) is assumed.

Figure 10 (on the left) shows $\langle\beta\rangle$ as a function of the heating power, $P$, for the three configurations with the scaled size. At the same power, $P$, the effect of the quite different neoclassical confinement is not as strong as would be expected from the ratio of monoenergetic transport coefficients in the $1 / \nu$-regime, which scale as $\epsilon_{\mathrm{eff}}^{3 / 2} / R^{2}$. The reason is that the radial electric field and different regimes of collisionality involved in the convolution procedure (4) lead to high nonlinearity of the thermal transport coefficients with respect to temperature and, as a result, diminish the large difference in transport implied by a "pure" $1 / \nu$-regime. On the other hand, however, the required power to achieve a given $\langle\beta\rangle$ reflects directly the very different degree of neoclassical confinement optimization. While for the W7-X configuration $\langle\beta\rangle \simeq 4 \%$ is obtained at $P=15 \mathrm{MW}$, the required $P$ strongly exceeds a realistic level for the optimized LHD configuration, and even $\langle\beta\rangle \simeq 2 \%$ is unreachable in the TJ-II configuration.

On the right of Fig. 10, $\tau_{E}$ from the predictive transport simulations normalized to the ISSO4 value is shown, which also reflects the neoclassical confinement improvement for the W7-X configuration and the degradation for the TJ-II case. The power degradation exponent in the ISS04 regression is $\alpha_{P}=-0.61$, but it must be noted that the simulation results do not follow this simple power-law dependence. In the range of $2 \mathrm{MW} \leq P \leq 10 \mathrm{MW}$, however, $\tau_{E}(P)$ is approximated in this form, leading to $\alpha_{P}=-0.64$ for TJ-II, $\alpha_{P}=-0.55$ for LHD, and $\alpha_{P}=-0.48$ for the W7-X configuration. These values can be compared with simple theory expectations: ${ }^{34} \alpha_{P}=-7 / 9$ for the $1 / \nu$-regime (with the most unfavorable temperature dependence of the transport coefficients), $\alpha_{P}=-3 / 5$ for the plateau regime (this is the Lackner-Gottardi scaling for tokamaks), $\alpha_{P}=0$ for transport coefficients independent of temperature, $\alpha_{P}=1 / 3$ for the stellarator $\sqrt{\nu}$-regime, and, finally, $\alpha_{P}=1$ in the tokamak banana regime. In case of the ion and electron energy fluxes being comparable but with both species in different transport regimes, $\tau_{E}$ cannot be expressed in the form of a simple scaling $\operatorname{law}^{34}$ (which is only possible if the particles dominating the total energy flux are in a pure transport regime).
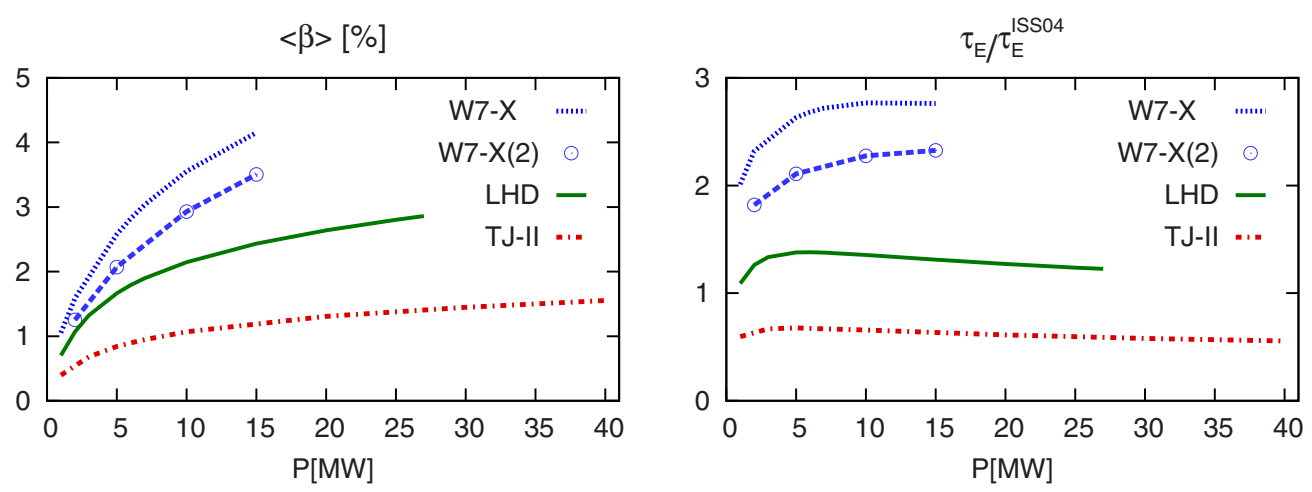

FIG. 10. (Color online) On the left, the volume-averaged plasma $\beta$ in W7-X, LHD, and TJ-II as a function of heating power; on the right, the energy confinement time normalized to the ISS04 scaling for W7-X, LHD, and TJ-II as a function of heating power; the curves marked as W7-X(2) are results of calculations performed similar to those presented by the W7-X curve but with the anomalous transport increased by a factor of 5 . 

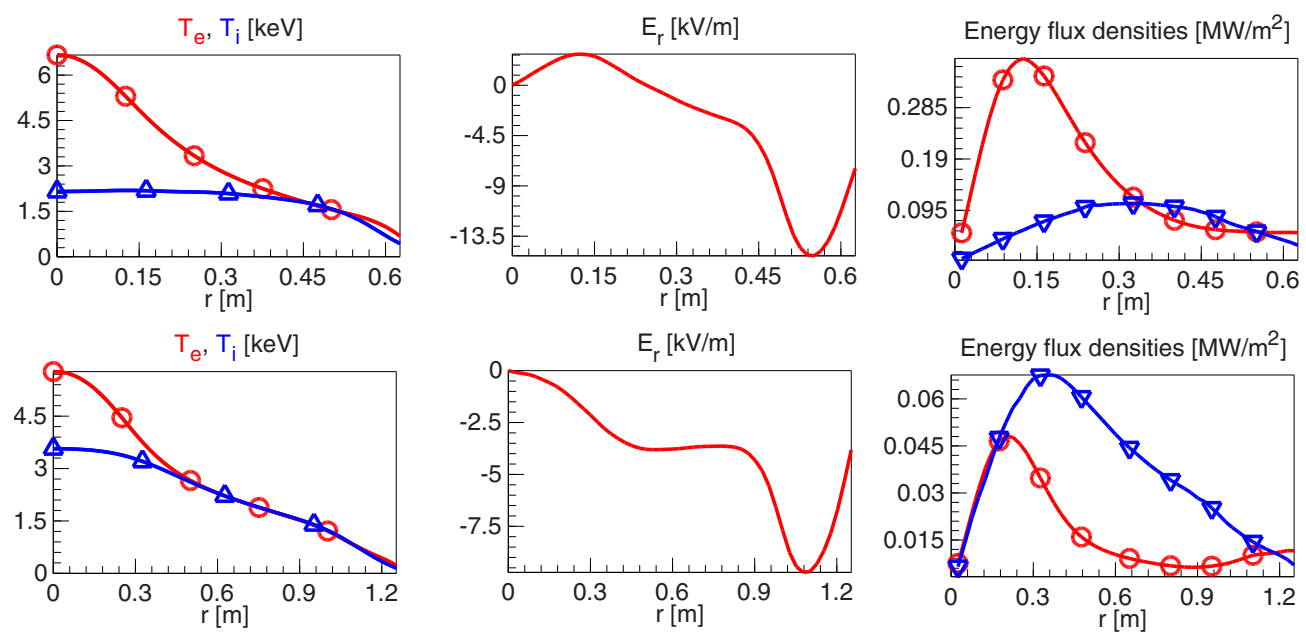

FIG. 11. (Color online) The temperatures, radial electric field, and energy fluxes in LHD for the case of 10 MW heating power; shown in the upper row are the results for the original device; in the lower row are the results for the same device with dimensions increased linearly by a factor of 2 ; electron temperatures and fluxes are shown by circles.

Finally, the impact of the anomalous energy diffusivities, $\chi_{e, i}$, is briefly discussed. Since no physics-based turbulent transport model is available for stellarators, the simplified form $\chi_{e, i} \propto 1 / n_{e}$ [as was indicated in W7-AS (Ref. 1)] is used inside the density gradient region (and further suppressed in the flat density region). Although experimentally indicated, ${ }^{35}$ a power dependence of $\chi_{e, i}$ is not taken into consideration. In the transport simulations of Fig. 10, an edge value of $\chi_{e}(a)$ $=\chi_{i}(a)=1.5 \mathrm{~m}^{2} / \mathrm{s}$ is assumed. For the W7-X scenarios, both anomalous transport coefficients $\chi$ are increased by a factor of 5 , leading to the reduction of $\langle\beta\rangle$ of about $15 \%$ [see the curves marked as W7-X(2) in Fig. 10]. The impact of the anomalous $\chi$ is also important for the $\mathrm{W} 7-\mathrm{X} \tau_{E}$ at very-low heating power where the neoclassical transport coefficients are significantly reduced due to the much lower temperature. Since the neoclassical transport coefficients, particularly at outer radii, are much higher in the LHD and TJ-II configurations, the anomalous transport modeling is less important for these two devices.

\section{Impact of device size on neoclassical confinement}

The monoenergetic neoclassical transport coefficients in the various collisionality regimes show a different dependence on the size [see Eq. (B2) in Appendix B). Consequently, the size dependence is briefly analyzed and compared with the ISS04 scaling in which $\tau_{E}$ increases roughly linearly with volume. In Fig. 11, transport simulations for the plasma density $n_{e}(0)=10^{20} \mathrm{~m}^{-3}$ and $10 \mathrm{MW}$ central heating are shown for the original LHD configuration (upper row) and increased linearly by a factor of 2 (lower row). The density and the heating power profiles [normalized to the corresponding plasma radius, see Fig. 1(left)] as well as the magnetic field strength are identical. An impact of the size on the anomalous energy diffusivity at the edge is disregarded. In the small size scenario, a stronger radial electric field is obtained (consistent with the stronger thermodynamic forces), which leads to slightly higher temperatures and $\langle\beta\rangle$ $=1.94 \%$ compared to the larger size case with $\langle\beta\rangle=1.73 \%$
( $\tau_{E}$ normalized to the ISS04 values is also reduced by nearly the same amount). Nearly the same results are obtained for a broader density profile in the larger configuration (where the density gradient region is reduced by a factor of 2).

The size scaling of the neoclassical transport coefficients is reflected by the different roles of electrons and ions in the energy flux densities. For a doubled size of the configuration, the electron contribution is roughly reduced by a factor of 4 , which is consistent with the expectation for the $1 / \nu$-regime in Eq. (B2). The ion energy transport coefficients are increased, although the size scaling for fixed $E_{r}$ in a pure $\sqrt{\nu}$-regime would lead to a reduction $\left(\propto f_{s}^{-1 / 2}\right)$, which, however, is overcompensated by the smaller radial electric field $\left(\propto E_{r}^{-3 / 2}\right)$. Consequently, the ions dominate the energy flux in this scenario. With increasing heating power, the electron energy transport coefficients increase significantly (as they have the most unfavorable $T_{e}$ dependence) and the electron contribution to the energy flux approaches the ion one. Rather equivalent results are also obtained for the W7-X configuration scaled in size; however, the degradation effect with respect to size is even larger: $\langle\beta\rangle=3.54 \%$ for the original size and $\langle\beta\rangle=2.92 \%$ for the configuration with the doubled size at $10 \mathrm{MW}$.

\section{CONCLUSIONS}

The energy balance solved by the predictive transport code based on the databases of the neoclassical monoenergetic transport coefficients precomputed with the DKES code $^{11,12}$ is benchmarked with the energy transport analysis with the DCOM database ${ }^{14,15}$ for an optimized LHD configuration as well as with the MOCA database ${ }^{16}$ for the standard TJ-II configuration. For both the thermal transport coefficients and the ambipolar radial electric field, very good agreement is obtained.

The energy confinement time of these simulations together with a W7-AS and a W7-X reference case is compared with the ISSO $4 \tau_{E^{-}}$scaling ${ }^{3}$ based on the experimental results of quite different stellarators. Again, very reasonable 
agreement is found, supporting the conclusion of the energy balance analyses at W7-AS (Ref. 1) that high-performance discharges with sufficiently high temperatures are dominated by neoclassical transport in the bulk part of the plasma; anomalous transport contributions are only dominant at the outer radii. In the ISS04 database, however, most discharges, particularly those with low temperature and high density, are not consistent with neoclassical transport modeling. The analysis of high-performance discharges, particularly at LHD, with respect to the impact of neoclassical energy confinement will be continued.

The database of monoenergetic neoclassical transport coefficients can be scaled with respect to an arbitrary size of the configuration. This procedure allows for a direct comparison of the different neoclassical confinement optimizations without using an empirical scaling law. Both the LHD and the TJ-II configurations are scaled in plasma volume to the W7-X standard configuration. For the same density and magnetic field strength, the volume-averaged $\langle\beta\rangle$ as well as $\tau_{E}$ normalized to the ISS04 scaling are calculated in a heating power scan. Although the monoenergetic transport coefficient (in particular, in the $1 / \nu$-regime, where it $\propto \epsilon_{\mathrm{eff}}^{3 / 2} / R^{2}$ ) differs by more than an order of magnitude, the difference in the obtained $\langle\beta\rangle$ and $\tau_{E}$ is significantly reduced due to the very strong nonlinearity of the thermal transport coefficients with respect to temperature. For fixed heating power, $\langle\beta\rangle$ is highest for the W7-X configuration, reduced by a factor of 2 for the LHD case, and further reduced by the same factor for the TJ-II configuration. Thereby, the degree of neoclassical optimization is directly reflected by the amount of heating power needed to obtain a given $\langle\beta\rangle$. Furthermore, the power degradation in $\tau_{E}$ is in reasonable agreement with the ISS04 scaling, although somewhat stronger for the TJ-II case and decreasing for the LHD and (even more) for the W7-X scenario.

Finally, the impact of the size scaling on the neoclassical confinement is consistent with the ISS04 scaling where $\tau_{E}$ roughly scales with the plasma volume. At fixed heating power, $\langle\beta\rangle$ is only slightly reduced for increased configuration size. While the electron heat transport is significantly decreased in the $1 / \nu$-regime with size, the ion heat transport is increased. For ions in the $\sqrt{\nu}$-regime, the decreased ambipolar radial electric field overcompensates the moderate reduction in the size scaling in the transport coefficients. Together with the density and the magnetic field strength dependence, the moderate size degradation in the neoclassical confinement needs further investigations.

A significantly improved neoclassical confinement allows for high temperatures and high plasma pressure. Increasing the magnetic field strength to reduce neoclassical transport leads to much greater forces on the coils and correspondingly greater demand on the support structure. Increasing only the size of a device does not help without increasing significantly the heating power. Consequently, neoclassical confinement must be improved to allow for a reasonable reactor perspective of stellarators. For an integrated reactor concept, however, additional constraints can be important. For example, an island divertor concept, which is based on the control of the edge island structure, requires the control of the plasma current and leads to the constraint of minimizing the bootstrap current. Consequently, the improvement in neoclassical transport is only one goal in the stellarator optimization procedure, but a very essential one. Otherwise, high temperatures and high plasma pressure (being important to confine $\alpha$-particles) cannot be achieved at a reasonable level of heating power.

\section{ACKNOWLEDGMENTS}

The authors acknowledge the helpful discussions with P. Helander. The work of V. Tribaldos was supported by Spain project MICINN ENE 2009-12213-C03-03/FTN.

\section{APPENDIX A: ESTIMATION OF AMBIPOLAR RADIAL ELECTRIC FIELD}

In stellarators, the neoclassical transport coefficients depend on $E_{r}$ and multiple roots of the ambipolarity condition can exist. In the $1 \mathrm{mfp}$ regime, typically three roots can appear: ${ }^{36,37}$ the ion root with small (usually negative) $E_{r}$, the electron root with strongly positive $E_{r}$, and an unstable root in between. In particular, in ECRH discharges with highly peaked power deposition, this feature has been experimentally identified in different devices. ${ }^{38}$ A problem occurs if these roots exist in a broader radial range and the position of the transition from the inner electron root to the outer ion root becomes important for predictive neoclassical transport simulations.

With a purely local neoclassical ordering scheme, the poloidal shear viscosity related to the transition in the radial electric field cannot be estimated since it depends on the finite radial deviations of particle orbits from the flux surface. ${ }^{23,25}$ From a thermodynamic point of view, however, the generalized heat production, ${ }^{20}$ which is the combination of the dissipation of the rotation energy in the transition region and the heat production rate of the neoclassical transport, must be minimized

$$
Q=\int_{0}^{a}\left\{\frac{D_{E}}{2}\left(E_{r}^{\prime}-\frac{E_{r}}{r}\right)^{2}+\frac{|e|}{\epsilon} \int^{E_{r}}\left(Z_{i} \Gamma_{i}-\Gamma_{e}\right) \mathrm{d} E_{r}\right\} V^{\prime} \mathrm{d} r .
$$

The Euler-Lagrange form of this variational problem leads to the diffusion equation (7) for the radial electric field. Here, $D_{E}$ is a formal diffusion coefficient (more strictly, $B^{2} D_{E}$ corresponds to a shear viscosity coefficient), which is adjusted in the predictive simulations for a reasonable size of the transition layer (the broadness of this layer scales with $D_{E}^{1 / 2}$ ). Solving this diffusion equation, a unique solution for $E_{r}$ is obtained, which smoothens the transition with respect to root-finding combined with an equivalent thermodynamic selection criterion.

With the assumption of a very narrow transition layer, the criterion for the position is obtained ${ }^{22}$ from the variation of the generalized heat production with respect to the radial position, 

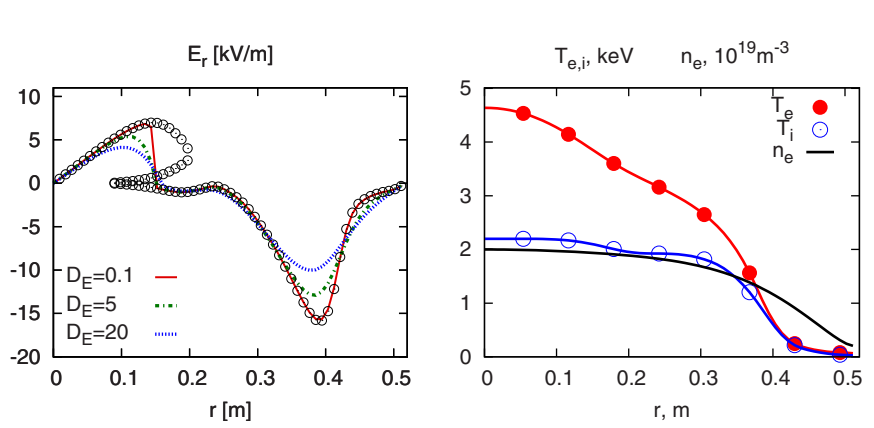

FIG. 12. (Color online) On the left, the ambipolar radial electric field; open circles are the solutions of the ambipolar condition (6); shown by lines are the solutions of the differential equation (7) for the different values of the diffusion coefficient. On the right, the plasma profiles used for solving ambipolar problem.

$$
\int_{E_{r}^{i}}^{E_{r}^{e}}\left(Z_{i} \Gamma_{i}-\Gamma_{e}\right) d E_{r}=0
$$

where $E_{r}^{i}$ and $E_{r}^{e}$ are the ion root and the electron root $E_{r}$, respectively. If this integral is positive (negative), the ion root (electron root) is realized. This criterion represents a local Maxwell constraint, which must be combined with the root-finding from the ambipolarity condition. The solution of the diffusion equation for $E_{r}$ adds only an additional equation to the set of balance equations being solved in the predictive transport simulations (with the same thermal transport coefficients). On the other hand, root-finding is strictly local (no information from the close vicinity enters) and, in principle, the existence of multiple roots must be checked for at all radii. Furthermore, the integral criterion must be evaluated if several roots are found leading to additional numerical effort. Consequently, the solution of the $E_{r}$ diffusion equation has strong advantages in predictive transport simulations. A disadvantage, however, appears if $E_{r}$ becomes very small in the transition region (this never appears in root-finding: $E_{r}$ is discontinuous) and where the ion transport coefficients can become very large as they enter the $1 / \nu$-regime, where the local neoclassical ordering scheme for ions is violated. Then, the ion temperature gradient (or more generally, the ion thermodynamic forces) must be strongly reduced to compensate for the peaked ion transport coefficients. Finally, a continuous $E_{r}$ within the transition region was experimentally identified from the heavy ion beam probe (HIBP) measurements at the Compact Helical System (CHS) (Ref 39). The radial electric fields calculated using different approaches are shown in Fig. 12. For the small diffusion coefficient, the differential equation (7) gives the same position of the transition from the electron root to ion root branch as obtained from Eq. (A2). A increase of the diffusion coefficient in Eq. (7) leads to broadening of the transition layer and a rather strong deviation of the solution from that obtained from the root-finding of Eq. (6) in the regions where the first derivative of the radial electric field changes rapidly. The plasma profiles used for solving this particular ambipolar problem are shown in the right portion of Fig. 12. They have been produced by predictive transport modeling of a plasma discharge in the W7-X standard magnetic configuration with $800 \mathrm{~kW}$ of ECRH.

\section{APPENDIX B: SCALING OF MONOENERGETIC TRANSPORT COEFFICIENTS}

The starting point is the linearized drift-kinetic equation (DKE), which becomes inhomogeneous with a radial driving force, $-\dot{r} F_{M}^{\prime}$ ( $r$ being the radius, $\dot{r}$ the radial component of the $\nabla B$-drift velocity, and $F_{M}^{\prime}$ the radial derivative of the Maxwellian with total energy conserved), and with a parallel driving force, $\propto v_{\|} B F_{M}$ (see, e.g., Ref. 9). Splitting this DKE with respect to the thermodynamic forces $A_{1}, A_{2}$ (both radial), and $A_{3}$ (parallel) leads to two first-order distribution functions, $f$ and $g$, where $f$ is related to $-\dot{r} F_{M}^{\prime}$ (symmetric in $\left.v_{\|}\right)$and $g$ to $v_{\|} B F_{M}$. For the treatment of the monoenergetic transport coefficients, both the thermodynamic forces and the Maxwellian are dropped in the following by introducing the definitions $f=\hat{f}\left(A_{1}+x^{2} A_{2}\right) F_{M}$ with $x=v / v_{\text {th }}$ and $g=\hat{g} A_{3} F_{M}$, where $\hat{f}$ and $\hat{g}$ are the solutions of the simplified DKE:

$$
\hat{\mathcal{V}}(\hat{f})-\hat{\nu} \mathcal{L}(\hat{f})=-\frac{R}{v} \dot{r} \quad \text { and } \quad \hat{\mathcal{V}}(\hat{g})-\hat{\nu} \mathcal{L}(\hat{g})=p b R
$$

with the "collisionality," $\hat{\nu}=\nu R / v$, the collision frequency $\nu(v)$, the major radius, $R$, and the normalized magnetic field strength, $b=B / B_{0}$. The radial component of the $\nabla B$-drift is given by

$$
R \dot{r}=\frac{T}{q} \frac{1+p^{2}}{B^{3}} x^{2}(\mathbf{B} \times \hat{\nabla} B)_{r}
$$

where $\hat{\nabla}=R \boldsymbol{\nabla}$ is the normalized gradient. $\hat{\mathcal{V}}$ is the (local and monoenergetic) normalized Vlasov operator,

$$
\hat{\mathcal{V}}=\left(\frac{p}{B} \mathbf{B}+\frac{\mathbf{E} \times \mathbf{B}}{v\left\langle B^{2}\right\rangle}\right) \cdot \hat{\nabla}_{\mathbf{s}}-\frac{1-p^{2}}{2 B^{2}} \mathbf{B} \cdot \hat{\nabla} B \frac{\partial}{\partial p},
$$

where $\mathbf{E}$ is the radial electric field, $\langle\cdots\rangle$ is the flux-surface average, and $\hat{\nabla}_{\mathbf{s}}$ is the normalized gradient within the flux surface. In Eq. (B1), $\mathcal{L}$ is the Lorentz form of the pitch-angle collision operator,

$$
\mathcal{L}=\frac{1}{2} \frac{\partial}{\partial p}\left(1-p^{2}\right) \frac{\partial}{\partial p} .
$$

The (traditional) monoenergetic transport coefficients calculated by DKES are defined by the following moments with $[A]=\int_{-1}^{1}\langle A\rangle d p / 2: D_{11}=[\dot{r} \hat{f}]$ is the particle diffusion coefficient, $D_{31}=v[b p \hat{f}]$ is the bootstrap current coefficient, $D_{13}=[\dot{r} \hat{g}]$ is the Ware pinch coefficient, and $D_{33}=v[b p \hat{g}]$ is the electric conductivity coefficient. Onsager symmetry leads to the relation $D_{31}=-D_{13}$.

The scaling of the monoenergetic transport coefficients with respect to the size of the device is performed by introducing the scaling factor defined by $f_{s}=R_{n} / R_{o}$, where $R_{n}$ is the new major radius and $R_{o}$ is the original one for which the DKES database was established. In the DKE (B1), both operators $\hat{\mathcal{V}}$ and $\mathcal{L}$ as well as $R \dot{r}$ are independent of $f_{s}$, and $\hat{g}$ scales with $f_{s}$ (in addition to the collisionality scaling). In the DKES database, the $D_{i j}$ coefficients are stored dependent on the radius, $r$, the "DKES collisionality," $\nu / v$, and the (normalized) radial electric field, $E_{r} / v B_{0}$, i.e., $D_{i j}\left(r, v / v, E_{r} / v B_{0}\right)$. The new "DKES collisionality" is simply scaled by $f_{s}$ for the 
interpolation in the original database. The electric field dependence of the $D_{i j}$ is not affected by the scaling factor $f_{s}$ and is omitted here for simplicity. With the new radii, $r$, the scaled new coefficients $D_{i j}^{n}$ are obtained from the original ones, $D_{i j}^{o}$, by

$$
\begin{aligned}
& D_{11}^{n}\left(r, \frac{\nu}{v}\right)=D_{11}^{o}\left(\frac{r}{f_{s}}, f_{s} \frac{\nu}{v}\right) / f_{s}, \\
& D_{13}^{n}\left(r, \frac{\nu}{v}\right)=D_{13}^{o}\left(\frac{r}{f_{s}}, f_{s} \frac{\nu}{v}\right), \\
& D_{31}^{n}\left(r, \frac{\nu}{v}\right)=D_{31}^{o}\left(\frac{r}{f_{s}}, f_{s} \frac{\nu}{v}\right), \quad D_{33}^{n}\left(r, \frac{\nu}{v}\right)=D_{33}^{o}\left(\frac{r}{f_{s}}, f_{s} \frac{\nu}{v}\right) f_{s} .
\end{aligned}
$$

Finally, some special cases of this size scaling are briefly analyzed. For the $1 / \nu$ regime, $D_{11} \propto f_{s}^{-2}$ is obtained, which recovers the analytic theory result. ${ }^{5}$ In the $\sqrt{\nu}$-regime, the impact of the size of the device is much less pronounced, $D_{11} \propto f_{s}^{-1 / 2}$, and in the plateau-regime, $D_{11} \propto f_{s}^{-1}$ is obtained. No size scaling of $D_{11}$ exists in all $\nu$-regimes. For the parallel conductivity coefficient, $D_{33} v / v$ is independent of the size in both the collisional and the collisionless limits.

${ }^{1}$ M. Hirsch, J. Baldzuhn, C. Beidler, R. Brakel, R. Burhenn, A. Dinklage, H. Ehmler, M. Endler, V. Erckmann, Y. Feng, J. Geiger, L. Giannone, G. Grieger, P. Grigull, H.-J. Hartfuß, D. Hartmann, R. Jaenicke, R. König, H. P. Laqua, H. Maaßberg, K. McCormick, F. Sardei, E. Speth, U. Stroth, F. Wagner, A. Weller, A. Werner, H. Wobig, S. Zoletnik, and W7-AS Team, Plasma Phys. Controlled Fusion 50, 053001 (2008).

${ }^{2}$ J. Baldzuhn, M. Kick, and H. Maßßberg, Plasma Phys. Controlled Fusion 40, 967 (1998).

${ }^{3}$ H. Yamada, J. H. Harris, A. Dinklage, E. Ascasibar, F. Sano, S. Okamura, J. Talmadge, U. Stroth, A. Kus, S. Murakami, M. Yokoyama, C. D. Beidler, V. Tribaldos, K. Y. Watanabe, and Y. Suzuki, Nucl. Fusion 45, 1684 (2005).

${ }^{4}$ C. D. Beidler, M. Yu. Isaev, S. V. Kasilov, W. Kernbichler, H. Maaßberg, D. R. Mikkelsen, S. Murakami, V. V. Nemov, M. Schmidt, D. A. Spong, V. Tribaldos, and A. Wakasa, Proceedings of the 22nd IAEA Fusion Energy Conference, Geneva, Switzerland, 2008.

${ }^{5}$ C. D. Beidler, K. Allmaier, M. Yu. Isaev, S. V. Kasilov, W. Kernbichler, G. O. Leitold, H. Maaßberg, D. R. Mikkelsen, S. Murakami, M. Schmidt, D. A. Spong, V. Tribaldos, and A. Wakasa, "Benchmarking of the monoenergetic transport coefficients-results from the international collaboration on neoclassical transport in stellarators (ICNTS)," Nucl. Fusion (submitted).

${ }^{6}$ M. Taguchi, Phys. Fluids B 4, 3638 (1992).

${ }^{7}$ H. Sugama and S. Nishimura, Phys. Plasmas 9, 4637 (2002).

${ }^{8}$ H. Sugama and S. Nishimura, Phys. Plasmas 15, 042502 (2008).

${ }^{9}$ H. Maaßberg, C. D. Beidler, and Y. Turkin, Phys. Plasmas 16, 072504 (2009).

${ }^{10}$ Yu. Turkin, H. Maaßberg, C. D. Beidler, J. Geiger, and N. B. Marushchenko, Fusion Sci. Technol. 50, 387 (2006).

${ }^{11}$ S. P. Hirshman, K. C. Shaing, W. I. van Rij, C. O. Beasley, Jr., and E. C. Crume, Phys. Fluids 29, 2951 (1986).

${ }^{12}$ W. I. van Rij and S. P. Hirshman, Phys. Fluids B 1, 563 (1989).

${ }^{13}$ A. Wakasa and S. Murakami, J. Plasma Fusion Res. 3, S1030 (2008).

${ }^{14}$ A. Wakasa, S. Murakami, H. Maßßerg, C. D. Beidler, N. Nakajima, K. Watanabe, H. Yamada, M. Okamoto, S. Oikawa, and M. Itagaki, J. Plasma
Fusion Res. 4, 408 (2001).

${ }^{15}$ S. Murakami, A. Wakasa, H. Maaßberg, C.D. Beidler, H. Yamada, and LHD Experimental Group, Nucl. Fusion 42, L19 (2002).

${ }^{16}$ V. Tribaldos, Phys. Plasmas 8, 1229 (2001).

${ }^{17}$ C. D. Beidler and W. N. G. Hitchon, Plasma Phys. Controlled Fusion 36, 317 (1994).

${ }^{18}$ V. V. Nemov, S. V. Kasilov, W. Kernbichler, and M. F. Heyn, Phys. Plasmas 6, 4622 (1999).

${ }^{19}$ C. D. Beidler and W. D. D'haeseleer, Plasma Phys. Controlled Fusion 37, 463 (1995).

${ }^{20}$ K. C. Shaing, Phys. Fluids 27, 1567 (1984).

${ }^{21}$ H. Maaßberg and K. S. Dyabilin, Proceedings of the 20th EPS Conference on Controlled Fusion Plasma Physics, Lisboa, 1993, Vol. 17C I, p. 409.

${ }^{22}$ H. Maassberg, R. Burhenn, U. Gasparino, G. Kühner, H. Ringler, and K. S. Dyabilin, Phys. Fluids B 5, 3627 (1993).

${ }^{23}$ D. E. Hastings, Phys. Fluids 28, 334 (1985).

${ }^{24}$ M. Sato, S. Toda, Y. Nakamura, K. Watanabe, A. Fukuyama, S. Murakami, M. Yokoyama, H. Funaba, H. Yamada, and N. Nakajima, J. Plasma Fusion Res. 3, S1063 (2008).

${ }^{25}$ D. E. Hastings, W. A. Houlberg, and K. C. Shaing, Nucl. Fusion 25, 445 (1985).

${ }^{26}$ Y. Turkin, C. D. Beidler, V. Erckmann, J. Geiger, H. P. Laqua, H. Maaßberg, N. B. Marushchenko, and W7-X Team, Proceedings of the 17th International Toki Conference and 16th International Stellarator/Heliotron Workshop, Toki, 2007, Paper No. P2-064.

${ }^{27}$ N. B. Marushchenko, H. Maaßberg, C. D. Beidler, V. Erckmann, J. Geiger, H. P. Laqua, A. G. Shalashov, E. V. Suvorov, Y. Turkin, and W7-X Team, in Proceedings of the Seventh International Workshop, 2008, edited by A. G. Litvak (Russian Academy of Science, Nizhny Novgorod, 2009), Vol. 2, p. 375.

${ }^{28}$ K. Uo, J. Phys. Soc. Jpn. 16, 1380 (1961).

${ }^{29}$ S. Murakami, H. Yamada, A. Wakasa, H. Inagaki, K. Tanaka, K. Narihara, S. Kubo, T. Shimozuma, H. Funaba, J. Miyazawa, S. Morita, K. Ida, S. Sakakibara, K. Y. Watanabe, M. Yokoyama, H. Maassberg, C. D. Beidler, and LHD Experimental Group, Fusion Sci. Technol. 51, 112 (2007).

${ }^{30}$ H. E. Mynick, T. K. Chu, and A. H. Boozer, Phys. Rev. Lett. 48, 322 (1982).

${ }^{31}$ V. Tribaldos and J. Guasp, Plasma Phys. Controlled Fusion 47, 545 (2005).

${ }^{32}$ B. A. Carreras, G. Grieger, J. H. Harris, J. L. Johnson, J. F. Lyon, O. Motojima, F. Rau, H. Renner, J. A. Rome, K. Uo, M. Wakatani, and H. Wobig, Nucl. Fusion 28, 1613 (1988).

${ }^{33}$ G. Grieger, W. Lotz, P. Merkel, J. Nührenberg, J. Sapper, E. Strumberger, H. Wobig, R. Burhenn, V. Erckmann, U. Gasparino, L. Giannone, H.J. Hartfuss, R. Jaenicke, G. Kühner, H. Ringler, A. Weller, and F. Wagner, Phys. Fluids B 4, 2081 (1992).

${ }^{34}$ A. Dinklage, H. Maßßberg, R. Preuss, Yu. A. Turkin, H. Yamada, E. Ascasibar, C. D. Beidler, H. Funaba, J. H. Harris, A. Kus, S. Murakami, S. Okamura, F. Sano, U. Stroth, Y. Suzuki, J. Talmadge, V. Tribaldos, K. Y. Watanabe, A. Werner, A. Weller, and M. Yokoyama, Nucl. Fusion 47, 1265 (2007).

${ }^{35}$ H. Ringler, U. Gasparino, G. Kühner, H. Maaßberg, H. Renner, and F. Sardei, Plasma Phys. Controlled Fusion 32, 933 (1990).

${ }^{36}$ H. E. Mynick and W. N. G. Hitchon, Nucl. Fusion 23, 1053 (1983).

${ }^{37}$ K. C. Shaing, R. H. Fowler, D. E. Hastings, W. A. Houlberg, E. F. Jaeger, J. F. Lyon, J. A. Rome, J. S. Tolliver, and J. D. Callen, Plasma Phys. Controlled Nucl. Fusion Res. 2, 189 (1984).

${ }^{38}$ M. Yokoyama, H. Maßßberg, C. D. Beidler, V. Tribaldos, K. Ida, F. Castejón, T. Estrada, A. Fujisawa, T. Minami, T. Shimozuma, Y. Takeiri, J. Herranz, S. Murakami, and H. Yamada, Fusion Sci. Technol. 50, 327 (2006).

${ }^{39}$ A. Fujisawa, H. Iguchi, T. Minami, Y. Yoshimura, K. Tanaka, K. Itoh, H. Sanuki, S.-I. Itoh, M. Isobe, S. Nishimura, S. Okamura, K. Matsuoka, and M. Fujiwara, Plasma Phys. Controlled Fusion 42, A103 (2000). 